\title{
An inequality on derived length of a solvable group
}

\section{Zhengfei $\mathrm{Wu}^{1}$, Xianhe Zhao ${ }^{2^{*}}$ and Shirong $\mathrm{Li}^{3}$}

"Correspondence:

zhaoxianhe989@163.com

${ }^{2}$ College of Mathematics and Information Science, Henan Normal

University, Xinxiang, Henan 453007,

P.R. China

Full list of author information is

available at the end of the article

\section{Abstract}

Let $G$ be a finite solvable group. Write $\delta^{*}(G)$ for the number of conjugacy classes of non-abelian subgroups of $G$, and by $d(G)$ denote the length of derived subgroups. In this paper an upper bound of $d(G)$ is given in terms of $\delta^{*}(G)$.

MSC: 20D10; 20D20

Keywords: solvable group; non-abelian subgroup; derived length

\section{Introduction}

In this paper $G$ is a solvable group of finite order and let $d(G)$ denote the derived length of $G$. By $\delta(G)$ denote the number of conjugacy classes of non-cyclic subgroups of $G$. It can be proved (see [1, Theorem 4.2]) that

$$
d(G) \leq 2(\delta(G)-1)^{1 / 2}+1 .
$$

This gives an upper bound of the derived length of $G$. Note that this bound is not nice, for which we have an improvement in this note.

In this paper by $\delta^{*}(G)$ denote the number of conjugacy classes of non-abelian subgroups of $G$, which will replace $\delta(G)$.

Recall some information about a formation which is required in this note. A class $\mathcal{F}$ of finite groups is called a formation if $G \in \mathcal{F}$ and $N \unlhd G$ then $G / N \in \mathcal{F}$, and if $G / N_{i}$ (i= $1,2) \in \mathcal{F}$ then $G / N_{1} \cap N_{2} \in \mathcal{F}$. If, in addition, $G / \Phi(G) \in \mathcal{F}$ implies $G \in \mathcal{F}$, we say that $\mathcal{F}$ to be saturated. The class of all abelian groups is a formation but not saturated; the class of all nilpotent groups is a formation and saturated [2, 9.5 Formations].

Let $\mathcal{F}$ be a subgroup-closed formation. and let $G^{\mathcal{F}}$ be the $\mathcal{F}$-residual of $G$, that is,

$$
G^{\mathcal{F}}=\bigcap_{N: N \unlhd G, G / N \in \mathcal{F}}(N) .
$$

Define

$$
\begin{aligned}
& G=G^{\mathcal{F}^{0}}, \\
& G^{\mathcal{F}^{i}}=\left(G^{\mathcal{F}^{i-1}}\right)^{\mathcal{F}}, \quad i=1,2, \ldots
\end{aligned}
$$

Consider the series of characteristic (normal) subgroups of $G$ :

$$
G=G^{\mathcal{F}^{0}} \geq G^{\mathcal{F}^{1}} \geq G^{\mathcal{F}^{2}} \geq \cdots \geq G^{\mathcal{F}^{r}}=1 .
$$

@ 2015 Wu et al.; licensee Springer. This is an Open Access article distributed under the terms of the Creative Commons Attribution License (http://creativecommons.org/licenses/by/4.0), which permits unrestricted use, distribution, and reproduction in any medium, provided the original work is properly credited. 
Here, if $r$ satisfies $G^{\mathcal{F}^{r}}=1$ but $G^{\mathcal{F}^{(r-1)}} \neq 1$, we say that $r$ is the $\mathcal{F}$-length of $G$. As $G$ is solvable, $r$ must exist and $r \geq 1$.

Note that $G / G^{\mathcal{F}}$ and $G^{\mathcal{F}^{(r-1)}}$ both are in $\mathcal{F}$, and

$$
G^{\mathcal{F}^{i}} / G^{\mathcal{F}^{(i+1)}}>1, \quad i=0,1, \ldots, r-1
$$

Suppose that $r \geq 4$. Now, we are able to choose elements $x_{0}, x_{1}, \ldots, x_{r-4}$ in $G=$ $G^{\mathcal{F}^{0}}, G^{\mathcal{F}^{1}}, \ldots, G^{\mathcal{F}^{(r-4)}}$ satisfying the following condition:

$$
x_{i} \in G^{\mathcal{F}^{i}} \text { but } x_{i} \notin G^{\mathcal{F}^{(i+1)}}, i=0,1, \ldots, r-4
$$

Define subgroups as follows:

$$
M_{i, j}=\left\langle x_{i}\right\rangle G^{\mathcal{F} j}, \quad i=0,1, \ldots, r-4, i+2 \leq j \leq r-2 .
$$

Note that:

(1) In the definition of $M_{i, j}, j \geq i+2$ is required.

(2) For every $i \in\{0,1, \ldots, r-4\}$, the set $\left\{y_{i}: y_{i} \in G^{(i)}-G^{\mathcal{F}^{(i+1)}}\right\}$ is non-empty, the $\left(x_{0}, x_{1}, \ldots, x_{r-4}\right)$ may be replaced by $\left(y_{0}, y_{1}, \ldots, y_{r-4}\right)$ in the note.

(3) If for some $i, G^{(i)} / G^{(i+1)}$ is a non-abelian 2-group, we say that the $i$ is a $\lambda(G)$. The investigation of the non- $\mathcal{F}$-subgroups of $G$ is an interesting problem.

\section{Preliminaries}

In this section we list some known results which are needed in the sequel.

Lemma 1 For all possible $i$ and $j$ we have:

(1) All $M_{i, j}$ are subgroups of $G$.

(2) No $M_{i, j}$ is in $\mathcal{F}$.

Proof (1) As all $G^{\mathcal{F} j}$ are characteristic (normal) subgroups of $G$, all $M_{i, j}$ must be subgroups of $G$.

(2) As $G^{\mathcal{F}^{r}}=1$ but $G^{\mathcal{F}^{(r-1)}} \neq 1$, we can see that $G^{\mathcal{F}^{(r-2)}}$ is not in $\mathcal{F}$. Next, by the definition of $M_{i, j}$, we have $r-2 \geq j$, so $G^{\mathcal{F}^{(r-2)}} \leq G^{\mathcal{F}^{j}} \leq M_{i, j}$. As $\mathcal{F}$ is subgroup-closed, we conclude that $M_{i, j} \notin \mathcal{F}$.

In the following part $\mathcal{F}$ is assumed to be the class of all abelian groups. Then $G^{\mathcal{F}}=G^{\prime}$ and $G^{\mathcal{F}^{(i)}}=G^{(i)}$ for all $i$. Lemma 2 is valuable for the following proofs.

Lemma 2 Let $G$ be a nilpotent group. Then that $G / G^{\prime}$ is cyclic implies $G$ is cyclic.

Proof In a nilpotent group, the derived subgroup is contained in the Frattini subgroup [2, 5.2.16], so $G^{\prime} \leq \Phi(G)$ and hence $G / \Phi(G)$ is cyclic. Thus $G$ is cyclic.

Lemma 3 Suppose that $G$ is a nilpotent group. Then no two of $M_{i, j}$ for all possible $i$ and $j$ are conjugate in $G$.

Proof Assume the lemma is false. So that there exist $M_{i, j}$ with $j \geq r+2$ and $M_{i^{\prime}, j^{\prime}}$ with $j^{\prime} \geq r+2$ which are conjugate, that is, there exists a $y \in G$ such that $M_{i, j}^{y}=M_{i^{\prime}, j^{\prime}}$. By definition 
of $M_{i, j}$ we have $G^{(j)} \leq G^{(i)}$. Thus, we have $M_{i, j}\left(=\left\langle x_{i}\right\rangle G^{(j)}\right) \leq G^{(i)}$. It follows that

$$
M_{i^{\prime}, j^{\prime}}=M_{i, j}^{y} \leq\left(G^{(i)}\right)^{y}=G^{(i)}
$$

Hence $x_{i^{\prime}} \in G^{(i)}$. By the choice of $x_{i}$, we have $i^{\prime} \geq i$. Similarly, $i \geq i^{\prime}$. It follows that $i=i^{\prime}$ and $x_{i}=x_{i^{\prime}}$.

In order to finish the proof, we also claim that $j=j^{\prime}$. Suppose that $j \neq j^{\prime}$. Without loss of generality, let $j<j^{\prime}$. Then $j+1 \leq j^{\prime}$ and

$$
\begin{aligned}
M_{i, j} & =\left\langle x_{i}\right\rangle G^{(j)} \geq\left\langle x_{i}\right\rangle G^{(j+1)} \\
& \geq\left\langle x_{i}\right\rangle G^{\left(j^{\prime}\right)}=\left\langle x_{i}^{\prime}\right\rangle G^{\left(j^{\prime}\right)}=M_{I^{\prime}, J^{\prime}} .
\end{aligned}
$$

We thus get

$$
\left\langle x_{i}\right\rangle G^{(j)}=\left\langle x_{i}\right\rangle G^{(j+1)} .
$$

Consequently, $G^{(j)} / G^{(j+1)}$ is cyclic.

Now, applying the hypothesis that $G$ is a nilpotent group, by Lemma 2 we find that $G^{(j)}$ is cyclic, consequently $j \leq r-2$, which is a contradiction (see the definition of $M_{i, j}$ ).

Lemma 4 ([1, Lemma 4.1]) Let $G$ be a solvable group. Then the following statements are true:

(1) $M_{i, 2 j}$ and $M_{i^{\prime}, 2 j^{\prime}}$ are conjugate if and only if $i=i^{\prime}$ and $j=j^{\prime}$.

(2) No $M_{i, j}$ is conjugate to some $G^{(k)}$.

Please note Lemmas 6 and 7 below.

Lemma 5 Let $G$ be a nilpotent group and $G^{(2)} \neq 1$. Then there exists a non-abelian subgroup $M$ which is a maximal subgroup in $G$, and the following statements are true:

(1) No subgroups $M_{i, j}$ for all possible $i$ and $j$ are conjugate to $M$.

(2) No subgroups $G^{(k)}$ for all possible $k$ are conjugate to $M$.

Proof By the condition that $G^{(2)} \neq 1$, so $G^{\prime}$ is non-abelian, and hence every maximal subgroup of $G$ which contains $G^{\prime}$ is non-abelian, for which we write $M$.

As $G$ is nilpotent, it follows that $G^{\prime} \leq \Phi(G)$ and hence $M$ is normal. Suppose some $M_{i, j}$ is conjugate to $M$. Then $M_{i, j}=\left\langle x_{i}\right\rangle G^{(j)}=M \unlhd G$ and $G^{\prime} \leq M=M_{i, j}$. If $x_{i} \in G^{\prime}$, as $G^{\prime}$ contains $G^{(j)}$, we see that $M_{i, j}=G^{\prime}<M<G$, which is a contradiction. Thus $x_{i} \notin G^{\prime}$, and it follows that $x_{i}=x_{0}$, and $M_{i, j}=M_{0, j}, j \geq 2$. Now, both $x_{0}$ and $G^{\prime \prime}$ are in $M_{0, j}$, hence $M_{0,2}=\left\langle x_{0}\right\rangle G^{\prime \prime}=M_{0, j}$. It follows that $M_{0,2} / G^{\prime \prime}$ is cyclic, by applying Lemma $2, G^{\prime}$ is cyclic, a contradiction.

Lemma 6 Let $G$ be a solvable group with $d(G)=r \geq 4$. Suppose that $G^{(i)} / G^{(i+1)}$ is a noncyclic 2-group for some fixed $i \in\{0,1, \ldots, r-4\}$. Fix this $i$ and take $a_{i}$ for an element of $G^{(i)}$ but not in $G^{(i+1)}$ and let $K_{i}=\left\langle a_{i}\right\rangle G^{(i+1)}$ (note that $\left.G^{(i)}>K_{i}>G^{(i+1)}\right)$. If $K_{i}$ is conjugate to some $M_{s, t}$ or some $G^{(k)}$, then $G^{(i)} / G^{(i+2)} \cong Q_{8}$, the quaternion group of order 8 .

Proof Fix $i$ and write

$$
G^{(i)} / G^{(i+1)}=\left\langle a_{1} G^{(i+1)}\right\rangle \times \cdots \times\left\langle a_{l} G^{(i+1)}\right\rangle,
$$


where all $a_{h}$ are 2-element, and $l \geq 2 . G^{(i)}$ is non-abelian, then $\left\langle a_{h}, G^{(i+1)}\right\rangle=\left\langle a_{h}\right\rangle G^{(i+1)}=K_{h}$ is non-abelian too.

As $G^{(i)}>K_{h}>G^{(i+1)}$, there is no $G^{(k)}$ which is conjugate to $K_{h}$. By condition, some $M_{s, t}$ is conjugate to $K_{h}$. Thus, for some $y \in G$ we have

$$
K_{h}=M_{s, t}^{y}=\left(\left\langle x_{s}\right\rangle G^{(t)}\right)^{y}=\left\langle x_{s}^{y}\right\rangle G^{(t)}, \quad t \geq i+2 .
$$

As $K_{h}<G^{(i)}$, it follows that

$$
\left\langle a_{h}\right\rangle G^{(i+1)}=K_{h}=\left\langle x_{s}^{y}\right\rangle G^{(t)}<G^{(i)} .
$$

Now, as $G^{(i+2)}<G^{(i+1)}<K_{h}$ when $i<r-4$, we have $G^{(i+2)}<G^{(i+1)}<K_{h}=M_{s, t}$, it follows that $K_{h} / G^{(i+2)}$ is a cyclic group of $M_{s, t} / G^{(i+2)}$ which is generated by $a_{h} G^{(i+2)}$. Hence $G^{(i+1)} / G^{(i+2)}$ is cyclic.

For any element $a$ of $G^{(i)}$ with order 2 (mould $G^{(i+2)}$ ), if $a \notin G^{(i+1)}$, then $\langle a\rangle \cap G^{(i+1)}=1$, contrary to $G^{(i+1)} / G^{(i+2)}$ being cyclic. Thus, $a \in G^{(i+1)}$ and it follows that $\langle a\rangle$ is a unique subgroup of order 2 (mould $G^{(i+2)}$ ), consequently $G^{(i)} \cong Q_{8}$ of order $8[2,5.3 .6]$, as desired.

Lemma 7 Let $G$ be a solvable group with $d(G)=r \geq 4$. Suppose that for some fixed $i \in\{0,1, \ldots, r-4\}, G^{(i)} / G^{(i+2)} \cong Q_{8}$ of order 8 . Then $G^{(i)}$ is non-nilpotent and contains an abnormal maximal subgroup $K$ which is non-abelian such that $K \in \delta^{*}(G)$.

Proof The condition that $d(G)=r \geq 4$ shows that $G^{(r-4)}$ is non-abelian. By the condition that $G^{(i)} / G^{(i+2)} \cong Q_{8}, G^{(i+1)} / G^{(i+2)}$ is cyclic of order 2, by Lemma 2 we see $G^{(i+1)}$ is cyclic, hence $G^{(i+2)}=1$. It follows that $r \leq i+2 \leq r-3+2=r-1$, a contradiction. Now we find that $G^{(i)}$ is non-nilpotent. Then there exists an abnormal maximal subgroup $K_{i}$ of $G^{(i)}$. If $K_{i}$ is abelian, then $G^{(r-3)} K_{i}=G$, this implies that $G^{\prime} \leq G^{(r-3)}$, contrary to $r \geq 4$. Now, we conclude that $K_{i}$ is non-abelian, as desired.

Obviously, no $G^{(s)}$ is conjugate to $K_{i}$ for all possible $s$. Suppose that some $M_{s, t}$ is conjugate to $K_{i}$. So, $M_{s, t}^{y}=K_{i}<G^{(i)}$ for some $y \in G$. By definition, $M_{s, t}=\left\langle x_{s}\right\rangle G^{(t)}$ with $t \geq s+2$. When $s \geq i+1$, then $x_{s}$ and $G^{(t)}$ both are in $G^{(i+1)}$, so $K_{i}=M_{s, t}^{y} \leq G^{(i+1)}=\left(G^{(i)}\right)^{\prime}$, contrary to $K_{i}$ being a maximal subgroup of $G^{(i)}$. Thus $s=i$ and $M_{s, t}=M_{i, t}$ with $t \geq i+2$. Now, $M_{s, t}=M_{i, t}=\left\langle x_{i}\right\rangle G^{(t)} \leq G^{(i)}$, so $G^{(i+2)} \geq G^{(t)}$. If $G^{(i+2)}>G^{(t)}$, we have $\left\langle x_{i}\right\rangle G^{(t)}>K$, consequently, $\left\langle x_{i}\right\rangle G^{(i+2)}=G^{(i)}$, and hence $\left\langle x_{i}\right\rangle G^{(i+1)}=G^{(i)}$, contrary to $Q_{8}\left(\cong G^{(i)} / G^{(i+2)}\right)$. Thus, $M_{s, t}=M_{i, i+2}=\left\langle x_{i}\right\rangle G^{(i+2)}$, which is normal in $G^{(i)}$, consequently, $K_{i}$ would be normal in $G^{(i)}$, a contradiction. We conclude that no $M_{s, t}$ is conjugate to $K_{i}$.

\section{Main results}

Now, we are able to give the main theorems of this note as follows:

Theorem 8 Let $G$ be a solvable group with $\delta^{*}(G) \geq 1$. Write $\lambda(G)=c+1$. Then

$$
d(G) \leq 2\left(\delta^{*}(G)-c-2\right)^{1 / 2}+1
$$

Proof In this section $\mathcal{F}$ denotes the class of all abelian groups, then $G^{\mathcal{F}}=G^{\prime}$ and $G^{\mathcal{F}^{i}}=$ $G^{(i)}$. If $d(G)=1,2,3$, then the theorem holds obviously. Let $d(G)=r \geq 4$. By Lemmas 1 and 3 , there exist the following non-abelian subgroups in $G$ : 
(a) $G\left(=G^{(0)}\right), G^{(1)}, G^{(2)}, \ldots, G^{(r-2)}$;

(b) $M_{0,2} ; M_{0,4}, M_{1,4}, M_{2,4} ; M_{0,6}, M_{1,6}, M_{2,6}, M_{3,6}, M_{4,6} ; \ldots$;

$$
M_{0,2 k}, M_{1,2 k}, \ldots, M_{2 k-2,2 k}, \quad r-2 \geq 2 k \geq r-3 .
$$

By Lemmas 6 and 7 for every $\lambda(G), G^{i_{l}}$ contains at least a non-abelian subgroup $K_{i_{l}}$, which belongs to $\delta^{*}(G)$, so we have

(c) $K_{i_{0}}, K_{i_{1}}, \ldots, K_{i_{c}}$.

No two of these subgroups are conjugate in $G$, therefore

$$
\begin{aligned}
\delta^{*}(G) & \geq(r-1)+(1+3+5+(2 k-1))+(c+1) \\
& =r+k^{2}+c \\
& \geq(r+c)+((r-3) / 2)^{2} .
\end{aligned}
$$

That is, $4 \delta^{*}(G) \geq(r-1)^{2}+4 c+9$,

$$
d(G) \leq 2\left(\delta^{*}(G)-c-2\right)^{1 / 2}+1
$$

In this case when $G$ is nilpotent, we have the following.

Theorem 9 Let $G$ be a nilpotent group with $d(G)=r$. Then

$$
d(G) \leq\left(2 \delta^{*}(G)+9 / 4\right)^{1 / 2}+1 / 2
$$

Proof If $d(G)=r=1,2,3$, then the theorem holds obviously. Let $d(G) \geq 4$. By Lemmas 1 and 4 , there exist the following non-abelian subgroups in $G$ :

(a) $G\left(=G^{(0)}\right), G^{(1)}, G^{(2)}, \ldots, G^{(r-2)}$;

(b) $M_{i, j}, i \in\{0,1, \ldots, r-4\}, r+2 \leq j \leq r-2$.

By Lemma 5 , every $G^{(i)}$ contains a non-abelian subgroup $K_{i}$ which is in $\delta^{*}(G)$, so we have

(c) $K_{0}, K_{1}, \ldots, K_{r-4}$.

No two of these subgroups are conjugate in $G$, therefore

$$
\begin{aligned}
\delta^{*}(G) & \geq(r-1)+(r-3)(r-2) / 2+(r-3) \\
& =(2 r-4)+(r-3)(r-2) / 2, \\
d(G) & \leq\left(2 \delta^{*}(G)+9 / 4\right)^{1 / 2}+1 / 2 .
\end{aligned}
$$

Example 10 Let $G=S_{4}$. Then $d(G)=3$ and $\delta^{*}(G)=4\left(S_{4}, S_{3}, A_{4}, Q_{8}\right)$.

(1) By Theorem 8, we have $(\lambda(G)=c+1=0)$

$$
2\left(\delta^{*}(G)-2-c\right)^{1 / 2}+1=2(4-1)^{1 / 2}+1<4.47 .
$$

We conclude that $d(G)=3<4.47$ and $4.47-r=1.47$. 
(2) $\delta(G)=5\left(S_{4}, S_{3}, A_{4}, Q_{8}, C_{2} \times C_{2}\right)$, by [1, Theorem 4.1], we have

$$
4.4 \leq 2(\delta(G)-1)^{1 / 2}+1=2(4)^{1 / 2}+1 \leq 5 .
$$

We conclude that $d(G)=3<5$ and $5-r=2$.

Competing interests

The authors declare that they have no competing interests.

Authors' contributions

All authors contributed equally and significantly in writing this article. All authors read and approved the final manuscript.

\section{Author details}

${ }^{1}$ School of Mathematics, South China University of Technology, Guangzhou, 510641, P.R. China. ${ }^{2}$ College of Mathematics and Information Science, Henan Normal University, Xinxiang, Henan 453007, P.R. China. ${ }^{3}$ Department of Mathematics, Guangxi University, Nanning, Guangxi 530004, P.R. China.

\section{Acknowledgements}

The authors are grateful to the editors and the referees, who provided their detailed reports. The research of the work was supported by NSFC (Grant Nos. 11171364, 11271301, U1204101, 11471266), Fundamental Research Funds for the Central Universities (No. XDJK2014C163), National Youth Science Foundation (No. 11201385) and the Major Project of Education Department of Henan Province (No. 13B110085)

Received: 20 October 2014 Accepted: 7 January 2015 Published online: 21 January 2015

\section{References}

1. Li, S, Zhao, X: Finite groups with few non-cyclic subgroups. J. Group Theory 10, 225-233 (2007)

2. Robinson, DJS: A Course in the Theory of Groups. Springer, New York (1982)

\section{Submit your manuscript to a SpringerOpen ${ }^{\odot}$ journal and benefit from:}

- Convenient online submission

- Rigorous peer review

- Immediate publication on acceptance

- Open access: articles freely available online

- High visibility within the field

- Retaining the copyright to your article 